\title{
Gravity as the Sole Fundamental Force
}

\author{
Gregory L. Light ${ }^{1}$ \\ ${ }^{1}$ Department of Finance, Providence College, 1 Cunningham Square, Providence, Rhode Island 02918, USA \\ Correspondence: Gregory L. Light, Department of Finance, Providence College, 1 Cunningham Square, Providence, \\ Rhode Island 02918, USA. E-mail: GLIGHT@ providence.edu
}

Received: March 16, 2019

Accepted: March 29, 2019

Online Published: April 4, 2019

doi:10.5539/apr.v11n3p23

URL: https://doi.org/10.5539/apr.v11n3p23

\begin{abstract}
:
We had explained electromagnetism by gravity before a recent publication in this Journal, in which we further incorporated the nuclear strong force in the framework of gravity. This paper, summarizing our cumulative results, continues to integrate the nuclear weak force with gravity, where we go by the following line of logic: Planck's formula shows energy $E=$ frequency $=$ probability $=$ wave; hence quantum waves have energies and the Universe is a diagonal spacetime manifold containing $\left\{\left(\right.\right.$ particle $p_{i}$, electromagnetic wave $\left.\left.\lambda\left(p_{i}\right)\right)\right\}$. By Feynman's analysis on electromagnetic mass, we assume that the distribution of $E$ over $(p, \lambda(p))$ is $\left(\frac{3}{4}, \frac{1}{4}\right) E$. Then Newton's gravitational acceleration formula yields $E=1.6 \times$ the observed energy of $p$, so that $p$ exists only for a duration of $\frac{5}{8} \frac{\lambda}{c}$ over the cycle $\left[0, \frac{\lambda}{c}\right]$, such as evidenced in quantum tunneling, opening the possibility for $\lambda(p)$ to be combined with other waves forming new particle(s) for $t>\frac{5}{8} \frac{\lambda}{c}$. By the time ratios of two frames in General Relativity we deduce neutron's lifetime, and by the Higgs mechanism we show neutron's decay products.
\end{abstract}

Keywords: neutron lifetime, beta decay, weak force by general relativity, unified field theory, hidden symmetry

\section{Introduction}

\subsection{Unfulfilled Goal of Unifying the Four Forces}

The quest for a unified field theory has been an ongoing endeavor in Physics for nearly a century (for a recent study, see Kamada, 2018). Previously we explained electromagnetism by an extended (set of) Einstein Field Equations

$$
R_{\mu \nu}-\frac{1}{2} g_{\mu \nu} R=-\frac{8 \pi G}{c^{2}} T \mu \nu, \operatorname{grav} \mp \frac{16 \pi G}{\left(1-\gamma^{-2} g_{11, \text { grav }}\right) c^{5}} T_{\mu \nu, e m}^{\text {att;rep }},
$$

and we invalidated the electroweak unification by noting the fact that in the Weinberg linear transformation an interchange of the two entries in (Higgs $\phi, 0)^{T}$ would fail the sought objective of distributing $\phi$ entirely to the $Z$ boson to leave photon without a rest mass.

\subsection{Hypothetical Background of Our Unification of the Four Forces}

We present a general description here (for analytical details, refer to Light, 2019): Before the Big Bang there had been $\mathcal{M}^{[2]}$ of electromagnetic waves (EMW's) without their particle presentations (now recognized as "dark energy") along with their collided standing masses (now recognized as "dark matter"). Because of the gravitational constant $G^{[2]} \approx 10^{85} G^{[3]}$ (the Newton constant), a cosmic black hole $\mathbf{B}$ formed in $\mathcal{M}^{[2]}$. The interior of $\mathbf{B}$ had (has) a quotient topology due to $g_{11}^{[2]}<0$. Since the center of mass of $\mathbf{B}$ had to be averaged over one common equivalence class of spacetime and the least class had length $10^{-63}$ meters < our assumed least indivisible unit of distance $10^{-35}$ meters, $\mathbf{B}$ blew up at its center (the Big Bang), creating (particle $p_{i}, E M W\left(p_{i}\right)$ ) existing in a diagonal spacetime manifold $\mathcal{M}^{[3]}=\mathcal{M}^{[1]} \times \mathbf{B}=$ the known Universe with linearization $(t+i t, x+i y, y+i z, z+i x)$, where $p_{i}=$ electron $e^{-}$, up quark $u$, down quark $d$, or neutrino $v$ along with its anti particle of the same handedness; the pair-creation came about through a pair of (photon $\gamma, E M W(\gamma))^{\prime} s$ intersecting each other at $90,60,30$, or 0 degrees respectively. For example, a left-handed electron $e_{L}^{-}$has its $E M W\left(e_{L}^{-}\right)$ spin

$$
\begin{aligned}
e_{L}^{-} & : \quad \text { from } W \equiv(-1,0,0) \text { to } N \equiv(0,1,0) \\
\text { to } E & \equiv(1,0,0) \text { to } T \equiv(0,0,1) \text { and back to } W,
\end{aligned}
$$

with

$$
e_{L}^{+}=E \rightarrow S \equiv(0,-1,0) \rightarrow W \rightarrow B \equiv(0,0,-1) \rightarrow E,
$$


as formed by

$$
\begin{aligned}
\gamma_{L}: & =W \rightarrow N \rightarrow E \rightarrow S \rightarrow W \text { and } \\
\gamma_{i L} & : \quad=E \rightarrow T \rightarrow W \rightarrow B \rightarrow E .
\end{aligned}
$$

A left-handed proton

$$
\begin{aligned}
P_{L}= & W \rightarrow T \rightarrow E \rightarrow N \rightarrow W \text { (opposite to } e_{L}^{-} \text {) } \\
& \text { or } W \rightarrow S \rightarrow E \rightarrow T \rightarrow W \text {, maintaining } 90^{\circ}
\end{aligned}
$$

$$
\begin{aligned}
& \text { Neutron } n \text { or } v \\
& =W \rightarrow N \rightarrow E \rightarrow N \rightarrow W \\
& \equiv E \rightarrow N \rightarrow W \rightarrow N \rightarrow E \\
& \equiv E \rightarrow T \rightarrow W \rightarrow T \rightarrow E ; \\
& \tilde{v}=W \rightarrow S \rightarrow E \rightarrow S \rightarrow W, \\
& \text { of opposite angular momentum to } v .
\end{aligned}
$$

(Without loss of generality, the following analysis will be presented by the left-handed convention.)

\section{Method and Results}

To make this paper self-contained yet without undue redundancy to our previous publication (Light, 2019), we will present the key logical elements of our integration of gravity with electromagnetism in Section 2.1 and that with the nuclear strong force in Section 2.2; we will then show our new results of integrating gravity with the nuclear weak force in Section 2.3 . Our method will be that of mathematical proofs with conclusions followed as results.

\subsection{Integration of Electromagnetism with Gravity}

Einstein Field Equations ("EFE")

$$
R_{\mu \nu}-\frac{1}{2} g_{\mu \nu} R=-\frac{8 \pi G}{c^{2}} T \mu \nu,
$$

begins with a Lorentzian metric $g$, which defines the associated covariant derivative $\nabla_{X} Y$ by the Koszul formula, then in turn the Riemann-Christoffel curvature $(1,3)$ - tensor $R(U, V) W$, in turn to the Ricci curvature $(0,2)-$ tensor $R_{\mu \nu}$ and finally to the Ricci curvature $(0,0)$-tensor $R$. That is, the entire left-hand-side $\mathcal{E}:=R i c-\frac{1}{2} g R$, recognized as the EinsteinHilbert tensor, is dependent on $g$ and is essentially the derivative of the total scalar curvature of a bounded manifold with respect to $g$. $E$ by its own nature is already an energy-momentum tensor $\mathcal{T}$, for which the constant of proportionality $\left(-\frac{8 \pi G}{c^{2}}\right)$ was (is) determined by Newton's gravitational law. The reason why using flat spacetime to determine physical constants is valid is owing to the algebraic homomorphism of tensors

$$
A^{*}(\alpha \mathbf{T})=\alpha A^{*} \mathbf{T}
$$

where $A^{*}$ is any pull-back operator of any tensor $\mathbf{T}$ that operates on vectors in a tangent space and $\alpha$ is any numerical constant; $A^{*}$ corresponds to any arbitrary frame transformation in physics, which includes in particular a frame situated in a flat spacetime. As such, $c, G$, and $h$ are universal, and all weak-field "approximations" (thus a misnomer) are to yield exact physical relationships.

Consider now the dynamics of a proton $Q\left(\equiv P\right.$ as denoted above) at $(0,0,0,0) \in U \subset \mathbb{R}^{1+3}$ (flat spacetime with Minkowski metric $\left.\eta:=\operatorname{diag}\left(1,-\frac{1}{c^{2}},-\frac{1}{c^{2}},-\frac{1}{c^{2}}\right)\right)$ that attracts an electron $q\left(\equiv e^{-}\right.$as denoted above $)$at $(0, x, y, z) \in U$; set

$$
r \equiv \sqrt{\left(x^{2}+y^{2}+z^{2}\right)}
$$

Proposition 1 Let $g$ be a local metric of a spacetime manifold $M^{4}$ and express $g$ as a matrix in the basis of $B \equiv$ $\left\{\frac{\partial f}{\partial t}, \frac{\partial f}{\partial x}, \frac{\partial f}{\partial y}, \frac{\partial f}{\partial z}\right\}$, where $f:(t, x, y, z) \in U \rightarrow(\tilde{t}, \tilde{x}, \tilde{y}, \tilde{z}) \in M^{4}$ is a local parametrization. Assume that $M^{4}$ is near flat; then a neighborhood $\tilde{U}$ of any point $\tilde{\mathbf{x}}=(\tilde{t}, \tilde{x}, \tilde{y}, \tilde{z}) \in M^{4}$ can be identified with the tangent space $T_{\tilde{\mathbf{x}}} \tilde{U}$, i.e., $\tilde{U} \equiv T_{\tilde{\mathbf{x}}} \tilde{U}$ with $\tilde{\mathbf{x}}=$ (i) a point in $\tilde{U}$ or (ii) a vector in $T_{\tilde{\mathbf{x}}} \tilde{U}$ (to be distinguished by a subscript "o" for clarity of the underlying context, such as a proper time $\tilde{t}_{o}$, which is the projection of the vector $(\tilde{t}, \tilde{x}, \tilde{y}, \tilde{z})$ onto the vector $(\tilde{t}, 0,0,0)$, but as a 1-dimensional value 
$\left.\tilde{t}_{o}:=\tilde{t}-\mathbf{0} \equiv \tilde{t}\right)$. Set the Lorentz transformation $L=f$; then the derivative of proper time $\tilde{t}_{o}$ with respect to proper time $t_{o}$ is:

$$
\frac{d \tilde{t}_{o}}{d t_{o}}=(1,0,0,0) g_{\mathbf{B}}\left(-1, v_{x}, v_{y}, v_{z}\right)^{T},
$$

where $\mathbf{V}=\left(v_{x}, v_{y}, v_{z}\right)$ is the velocity of $q$.

Proof. Without loss of generality, consider

$$
L=\gamma\left(\begin{array}{cc}
1 & \frac{v}{c^{2}} \\
v & 1
\end{array}\right)
$$

transforming frame $S$ to $\tilde{S}$, and calculate

$$
\begin{aligned}
& (1,0) g_{\mathbf{B}}(-1, v) \\
= & (1,0)\left(\left(L^{-1}\right)^{T}\right)^{-1}\left[\left(L^{-1}\right)^{T} g_{\mathbf{B}} L^{-1}\right] L(-1, v)^{T} \\
\approx & (1,0)\left(\gamma\left(\begin{array}{cc}
1 & v \\
\frac{v}{c^{2}} & 1
\end{array}\right)\right)\left(\begin{array}{cc}
1 & 0 \\
0 & -\frac{1}{c^{2}}
\end{array}\right)\left(\begin{array}{c}
\Delta \tilde{t}_{o} \\
0
\end{array}\right) \\
= & \left(\gamma,-\frac{\gamma v}{c^{2}}\right)\left(\begin{array}{c}
\Delta \tilde{t}_{o} \\
0
\end{array}\right)\left(\text { observe that } L:(-1, v)^{T} \longmapsto\left(\Delta \tilde{t}_{o}, 0\right)^{T},\right. \\
= & \frac{\Delta \tilde{t}_{o}}{\sqrt{1-\left(\frac{v}{c}\right)^{2}}}=\frac{\Delta \tilde{t}_{o} \text { is the proper time of } \tilde{S} \text { by definition) }}{\left\|(-1,-v)^{T}\right\|_{\eta}}=\frac{\Delta \tilde{t}_{o}}{\left\|L^{-1}(-1,-v)^{T}\right\|_{\eta}} \\
= & \frac{\Delta \tilde{t}_{o}}{\Delta t_{o}} \approx \frac{d \tilde{t}_{o}}{d t_{o}},\left(\text { where } L^{-1}:(-1,-v)^{T} \longmapsto\left(\Delta t_{o}, 0\right)^{T},\right. \\
& \text { analogous to the above Equation for } L) .
\end{aligned}
$$

Remark 1 The meaning of $\frac{d \tilde{t}_{o}}{d t_{o}}=(1,0,0,0) g_{\mathbf{B}}\left(-1, v_{x}, v_{y}, v_{z}\right)^{T}$ is:

$$
\begin{aligned}
\Delta t_{o} & \rightarrow\left(\frac{\Delta t_{o}}{\Delta t_{o}}, \frac{\Delta x}{\Delta t_{o}}, \frac{\Delta y}{\Delta t_{o}}, \frac{\Delta z}{\Delta t_{o}}\right)^{T} \in U \\
& =\left(1,-v_{x},-v_{y},-v_{z}\right)^{T} \text { (sign due to attractive action) } \\
& \rightarrow\left(1 \cdot \frac{\partial f}{\partial t}-v_{x} \frac{\partial f}{\partial x}-v_{y} \frac{\partial f}{\partial y}-v_{z} \frac{\partial f}{\partial z}\right)^{T} \in \tilde{U} \\
& \rightarrow g_{\mathbf{B}}\left(1 \cdot \frac{\partial f}{\partial t}-v_{x} \frac{\partial f}{\partial x}-v_{y} \frac{\partial f}{\partial y}-v_{z} \frac{\partial f}{\partial z}\right)^{T} \\
& =\left(1,-\tilde{v}_{x},-\tilde{v}_{y},-\tilde{v}_{z}\right)^{T}=\left(\frac{\Delta \tilde{t}_{o}}{\Delta \tilde{t}_{o}}, \frac{\Delta \tilde{x}}{\Delta \tilde{t}_{o}}, \frac{\Delta \tilde{y}}{\Delta \tilde{t}_{o}}, \frac{\Delta \tilde{z}}{\Delta \tilde{t}_{o}}\right)^{T} \\
& \rightarrow \Delta \tilde{t}_{o} .
\end{aligned}
$$

Remark 2 A geodesic from $\tilde{\mathbf{x}}_{1}$ to $\tilde{\mathbf{x}}_{2}$ equals

$$
\max _{\left\{\left(t=t_{o}, x(t), y(t), z(t)\right)\right\}} \int_{f^{-1}\left(\tilde{\mathbf{x}}_{1}\right)}^{f^{-1}\left(\tilde{\mathbf{x}}_{2}\right)} \frac{d \tilde{t}_{o}}{d t_{o}} d t_{o}
$$

and corresponds to the least action

$$
\begin{aligned}
& \min \int(\text { kinetic energy } K E-\text { potential energy } P E) d t \\
\Longleftrightarrow & \max \int\left(a+b\left(\frac{P E-K E}{\text { rest energy } R E}\right)\right) d t, a \in \mathbb{R}, b>0, \\
\Longleftrightarrow & \frac{d \tilde{t}_{o}}{d t_{o}}=a+b\left(\frac{P E-K E}{\text { rest energy } R E}\right), \text { by Equation (15), } \\
= & (1,0,0,0) g_{\mathbf{B}}\left(-1, v_{x}, v_{y}, v_{z}\right)^{T}, \text { by Proposition } 1 .
\end{aligned}
$$


I.e.,

$$
(1,0,0,0) g_{\mathbf{B}}\left(-1, v_{x}, v_{y}, v_{z}\right)^{T}=a+b\left(\frac{P E-K E}{\text { rest energy } R E}\right) .
$$

Remark 3 (1) $\frac{d \tilde{t}_{o}}{d t_{o}}=$ the effect of the velocity $V\left(t_{o}\right)$ of the electron $q$ in the electromagnetic field as established by $q$ and the proton $Q$ on $q^{\prime} s$ proper time. (2) $\left.\frac{\tilde{t}_{o}}{t_{o}}\right\rfloor_{t_{o}=t^{*}}=$ the eigenvalue of the Lorentz transformation $\lambda\left(t^{*}\right)=\gamma\left(t^{*}\right)\left(1-\frac{v\left(t^{*}\right)}{c}\right)=$ $\sqrt{\frac{c-v\left(t^{*}\right)}{c+v\left(t^{*}\right)}}=$ the ratio of the clock time of $q$ to that of $Q$ at $t_{o}=t^{*}$ ( $\lambda$ accounts for the Doppler effect of light). (3) The Lorentz factor $\gamma\left(t^{*}\right)=\frac{1}{\sqrt{1-\left(\frac{v\left(t^{*}\right)}{c}\right)^{2}}}=\frac{\text { the clock time of } q \text { at } t_{o} t^{*}}{\text { the universal time over } U \text { as synchronized with } t_{o}=t^{*} \text { of } Q}(\gamma$ accounts for time dilation $)$.

Proposition $2 \operatorname{Let} v(t):=\|\mathbf{V}(t)\|$ and $v_{Q}(t):=\left\|\mathbf{V}_{Q}(t)\right\| ;$ then

$$
\gamma^{2}\left(\frac{v(t)}{c}\right)=\frac{\text { the electric potential energy } P E \text { of } Q \text { and } q}{\text { the rest energy } R E \text { of } q} .
$$

Proof.

$$
\begin{aligned}
\left(\frac{v(t)}{c}\right) & =\left(\frac{1}{m_{q, o} c^{2}}\right) \cdot q\left(\frac{Q}{q} \frac{v_{Q}(t)}{c} \frac{r}{r_{o}}\right) \cdot \frac{Q}{4 \pi \epsilon_{o} r} \\
& \equiv \frac{1}{R E} \cdot K \cdot \frac{q Q}{4 \pi \epsilon_{o} r}
\end{aligned}
$$

where

$$
K \equiv \frac{Q}{q} \frac{v_{Q}(t)}{c} \frac{r}{r_{o}}=-\frac{v_{Q}(t) \cdot\left(\frac{r}{c}\right)}{r_{o}}
$$

is an electrodynamic adjustment factor of the electrostatic potential;

$$
K=-1 \text { if } v_{Q}(t) \cdot\left(\frac{r}{c}\right) \equiv v_{Q}(t) \cdot t=r_{o},
$$

i.e., the point charge $Q$ travels to the boundary of its wave ball $r_{o}$, or equivalently, $Q$ is a stationary proton. Thus, taking into account the effect of Special Relativity

$$
\mathbf{F}=\left(\gamma m_{o}\right)\left(\gamma^{2} \mathbf{a}\right)
$$

we have

$$
\gamma^{2}\left(\frac{v(t)}{c}\right)=\frac{\gamma^{2} K Q q / 4 \pi \epsilon_{o} r}{R E}=\frac{P E}{R E} .
$$

\section{Proposition 3}

$$
-\gamma^{ \pm 2}\left(\frac{v(t)}{c}\right)\left(\frac{v(t) v_{Q}(t)}{c^{2}}\right)=\frac{q \mathbf{V}(t) \cdot \mathbf{A}(t)}{R E},
$$

where $A(t):=$ the vector potential, or curl $A(t)=$ the magnetic field $B(t)$.

Proof. Since

$$
\begin{aligned}
-v(t) v_{Q}(t) & =\mathbf{V}(t) \cdot \mathbf{V}_{Q}(t) \text { and } \\
\frac{\gamma^{2} K Q \mathbf{V}_{Q}(t)}{4 \pi \epsilon_{o} r c^{2}} & =\mathbf{A}(t),
\end{aligned}
$$

we have

$$
\begin{aligned}
& -\gamma^{2}\left(\frac{v(t)}{c}\right)\left(\frac{v(t) v_{Q}(t)}{c^{2}}\right) \\
= & \frac{\gamma^{2} K Q q \mathbf{V}(t) \cdot \mathbf{V}_{Q}(t)}{R E \cdot 4 \pi \epsilon_{o} r c^{2}}=\frac{q \mathbf{V}(t) \cdot \mathbf{A}(t)}{R E} .
\end{aligned}
$$


Proposition 4 For any weakly attractive or repulsive electromagnetic field, the metric $g_{e m}^{\text {att; }}{ }^{\text {rep }}$ has the following matrix representation in the basis of $B$ (for generalization, we now include the repulsive case, shown as the second or lower index):

$$
g_{e m}^{\text {att; rep }}=\left(\begin{array}{cccc}
\lambda_{e m}^{ \pm 2} & -\frac{2 \gamma^{ \pm 2} v_{Q} \mathbf{V}_{x}}{c^{3}} & -\frac{2 \gamma^{ \pm 2} v_{Q} \mathbf{V}_{y}}{c^{3}} & -\frac{2 \gamma^{ \pm 2} v_{Q} \mathbf{V}_{z}}{c^{3}} \\
-\frac{2 \gamma^{ \pm 2} v_{Q} \mathbf{V}_{x}}{c^{3}} & o\left(\frac{v}{c}\right)-c^{-2} & o\left(\frac{v}{c}\right)^{3} & o\left(\frac{v}{c}\right)^{3} \\
-\frac{2 \gamma^{ \pm 2} v_{Q} \mathbf{V}_{y}}{c^{3}} & o\left(\frac{v}{c}\right)^{3} & o\left(\frac{v}{c}\right)-c^{-2} & o\left(\frac{v}{c}\right)^{3} \\
-\frac{2 \gamma^{ \pm 2} v_{Q} \mathbf{V}_{z}}{c^{3}} & o\left(\frac{v}{c}\right)^{3} & o\left(\frac{v}{c}\right)^{3} & o\left(\frac{v}{c}\right)-c^{-2}
\end{array}\right) .
$$

Proof. First, we note that besides being symmetric, $g_{e m}^{\text {att; } r e p} \longrightarrow \eta$ as $\mathbf{V}, \mathbf{V}_{Q} \longrightarrow \mathbf{0}$. Second,

$$
g_{11, e m}^{a t t ; r e p}=\lambda_{e m}^{ \pm 2}=\left(\frac{\tilde{t}_{o}}{t_{o}}\right)_{\text {att; rep }}^{2}(\text { cf. Remark } 3(2)) .
$$

Third, by Proposition 1 we have

$$
\begin{aligned}
\frac{d \tilde{t}_{o}}{d t_{o}} & =(1,0,0,0) g_{\mathbf{B}}\left(\mp 1, \mathbf{V}_{x}, \mathbf{V}_{y}, \mathbf{V}_{z}\right)^{T} \\
& =\mp \lambda^{ \pm 2}-\frac{2 \gamma^{ \pm 2} v_{Q} v^{2}}{c^{3}} \\
& \approx \mp \gamma^{ \pm 2}\left(1 \mp \frac{2 v}{c}\right)+\frac{2 q \mathbf{V} \cdot \mathbb{A}}{R E}(\text { by Proposition 3) } \\
& \approx \mp\left(1 \pm\left(\frac{v}{c}\right)^{2}\right)+\frac{2(P E+q \mathbf{V} \cdot \mathbb{A})}{R E}(\text { by Proposition 2) } \\
& =\mp 1-\frac{m_{o} v^{2}}{m_{o} c^{2}}+\frac{2\left(P E_{e}+q \mathbf{V} \cdot \mathbb{A}\right)}{R E} \\
& =\mp 1-\frac{2\left(\text { kinetic energy } K E-P E_{e}-q \mathbf{V} \cdot \mathbb{A}\right)}{R E} ;
\end{aligned}
$$

thus, by Equation (17) we see that $g_{e m}^{a t t ;}$ rep $=g_{\mathbf{B}}$, satisfying the least-action energy condition, and the theorem is proved.

Lemma 1 Assume that the energy-momentum tensor $T \approx 0$ for $M^{4}$; then

$$
R \approx 2 R_{11} \approx-\frac{6}{r_{K}^{2}},
$$

where $r_{K}>0$ denotes the radius of curvature of the space-time.

Proof. Let $U:=\left\{\left(x^{1} s, x^{2}\right.\right.$ im, $x^{3}$ im, $x^{4}$ im $\left.) \mid\left(x^{1}, x^{2}, x^{3}, x^{4}\right) \in \mathbb{R}^{4}\right\}$ be a parameter domain of $M^{4}$; adopt the Cartesian coordinate system on $U$; let $\left(\mathbf{e}_{i}\right)_{i=1}^{4}$ be the standard basis of $\mathbb{R}^{4}$; define metric $I$ to be the standard inner product of $\mathbb{R}^{4}$, i.e., $I:=\operatorname{diag}(1,1,1,1)_{\left(\mathbf{e}_{i}\right)_{i=1}^{4}}$. Since $T \approx \mathbf{0}$, the space-time is asymptotically flat and thus any Lorentzian metric $g$ on $M^{4}$ is such that $g \approx I$. Consequently,

$$
\begin{aligned}
R & \equiv R(g) \approx R(I) \\
& =R_{11}(I)+R_{22}(I)+R_{33}(I)+R_{44}(I) \\
& =\sum_{i=1}^{4} R_{1 i 1}^{i}(I)+\sum_{i=1}^{4} R_{2 i 2}^{i}(I)+\sum_{i=1}^{4} R_{3 i 3}^{i}(I)+\sum_{i=1}^{4} R_{4 i 4}^{i}(I) ;
\end{aligned}
$$

now $\forall i \neq j \in\{1,2,3,4\}$ every sectional curvature $\left|R_{i j i}^{j}(I)\right| \approx 0 s^{-2} \approx|K| s^{-2}$, where they fall into two categories: $(t, x)$ and $(x, x)$, i.e.,

$$
\begin{aligned}
\forall(i-1)(j-1) & =0, \\
\left|R_{i j i}^{j}\right| & \approx|K| s^{-2} \approx\left(3 \times 10^{8}\right)^{-1}|K| \cdot s^{-1} m^{-1} \\
\operatorname{but} \forall(i-1)(j-1) & \neq 0, \\
\left|R_{i j i}^{j}\right| & \approx|K| s^{-2} \approx\left(3 \times 10^{8}\right)^{-2}|K| \cdot m^{-2} \approx 0 m^{-2} .
\end{aligned}
$$


Thus,

$$
\begin{aligned}
R & \equiv R(g) \approx R(I) \approx R_{11}(I)+\left[R_{212}^{1}(I)+R_{313}^{1}(I)+R_{414}^{1}(I)\right] \\
& \approx 3 \cdot\left(3 \times 10^{8}\right)^{-1} K \cdot s^{-1} m^{-1}+\left[3 \cdot\left(3 \times 10^{8}\right)^{-1} K \cdot s^{-1} m^{-1}\right] \\
& =6 \cdot\left(3 \times 10^{8}\right)^{-1} K \cdot s^{-1} m^{-1} \\
& \approx 2 R_{11}(I) .
\end{aligned}
$$

Since metric $I$ is equivalent to the Minkowski metric

$$
\begin{aligned}
\eta & :=\operatorname{diag}\left(1 s^{2} / s^{2},-c^{-2} s^{2} / m^{2},-c^{-2} s^{2} / m^{2},-c^{-2} s^{2} / m^{2}\right)_{\left(\mathbf{e}_{i}\right)_{i=1}^{4}} \\
& =\operatorname{diag}\left(1,-c^{-2},-c^{-2},-c^{-2}\right)_{\left(\mathbf{e}_{i}\right)_{i=1}^{4}},
\end{aligned}
$$

we thus have

$$
\begin{aligned}
R_{11} & \equiv R_{11}(g) \approx R_{11}(\eta)=\eta^{11} R_{11}(\eta)=I^{11} R_{11}(I)=R_{11}(I) \\
\text { i.e., } R & \equiv R(g) \approx 2 R_{11}(I) \approx 2 R_{11} .
\end{aligned}
$$

Since $\eta$ measures space-time distance by $d s$ and

$$
d s^{2}=d t^{2}-c^{-2} d x^{2} \text { is hyperbolic, }
$$

we have for each space-time sectional curvature $\approx K<0$; denoting the radius of curvature by $r_{K}>0$, we then have

$$
\begin{aligned}
R & \approx 2 R_{11} \approx 6 \cdot\left(3 \times 10^{8}\right)^{-1} K \cdot s^{-1} m^{-1} \\
& \equiv-\frac{6}{r_{K}^{2}} m^{-2} .
\end{aligned}
$$

Proposition 5 The Einstein tensor

$$
\mathcal{E}_{e m}^{a t t ; r e p} \approx\left(\begin{array}{cccc}
\mp \frac{6 v}{r_{k}^{2} c} & -\frac{6 v_{Q} \mathbf{V}_{x}}{r_{k}^{2} c^{3}} & -\frac{6 v_{Q} \mathbf{V}_{y}}{r_{k}^{2} c^{3}} & -\frac{6 v_{Q} \mathbf{V}_{z}}{r_{k}^{2} c^{3}} \\
-\frac{6 v_{Q} \mathbf{V}_{x}}{r_{k}^{2} c^{3}} & -O\left(r_{k}^{-2}\right) & O\left(r_{k}^{-2} c^{-4}\right) & O\left(r_{k}^{-2} c^{-4}\right) \\
-\frac{6 v_{Q} \mathbf{V}_{y}}{r_{k}^{2} c^{3}} & O\left(r_{k}^{-2} c^{-4}\right) & -O\left(r_{k}^{-2}\right) & O\left(r_{k}^{-2} c^{-4}\right) \\
-\frac{6 v_{Q} \mathbf{V}_{z}}{r_{k}^{2} c^{3}} & O\left(r_{k}^{-2} c^{-4}\right) & O\left(r_{k}^{-2} c^{-4}\right) & -O\left(r_{k}^{-2}\right)
\end{array}\right)_{\mathbf{B}}
$$

Proof. $\mathcal{E}_{\mu v}:=R_{\mu \nu}-\frac{1}{2} R \cdot g_{\mu v} ; \forall \mathcal{M}^{4} \approx \mathbb{R}^{1+3}$ we have

$$
\begin{aligned}
\left(R_{\mu \nu}\right) & \approx \operatorname{diag}\left(-\frac{3}{r_{K}^{2}},-\frac{1}{r_{K}^{2}},-\frac{1}{r_{K}^{2}},-\frac{1}{r_{K}^{2}}\right) \text { and } \\
R & \approx-\frac{6}{r_{K}^{2}}
\end{aligned}
$$

(where $r_{K} \equiv$ the radius of sectional curvatures). Thus, substituting Equation (30) into $\left(g_{\mu v}\right)$ in $\left(\mathcal{E}_{\mu v}\right)$, we arrive at the conclusion.

\section{Proposition 6 Set}

$$
\begin{aligned}
& \mathcal{T}_{\text {em, }, 11}^{\text {att; } r e p}= \pm\|\mathbf{S}\|= \pm \frac{m_{o, Q} v_{Q}}{\frac{4 \pi r^{3}}{3}} \cdot c^{2} \text { and } \\
& \mathcal{T}_{\text {em,12 }}^{\text {att; rep }}=-\frac{\|\mathbf{S}\| v_{Q, x}}{c^{2}} \text {; then } \\
& \frac{\mathcal{E}_{e m, 12}^{a t t ; r e p}}{\mathcal{E}_{e m, 11}^{\text {att rep }}}=\frac{\mathcal{T}_{e m, 12}^{\text {att; rep }}}{\mathcal{T}_{e m, 11}^{\text {att;rep }}} .
\end{aligned}
$$


Proof.

$$
\begin{aligned}
\frac{\mathcal{E}_{12, \text { em }}}{\mathcal{E}_{11, \text { rem }}^{\text {att }}} & = \pm \frac{1}{c^{2}}\left(\frac{v_{Q}}{v}\right) V_{x}(\text { by Equation }(46)) \\
& = \pm \frac{1}{c^{2}} \cdot\left(-V_{Q, x}\right) \\
& =\frac{-\frac{\|\overline{\mathbf{S}}\|}{c^{2}} V_{Q, x}}{ \pm\|\overline{\mathbf{S}}\|} \equiv \frac{\mathcal{T}_{12, e m}}{\mathcal{T}_{11, \text { em }}^{\text {att rep }}}(\text { by Equation }(50))
\end{aligned}
$$

\section{Proposition 7}

$$
\frac{\mathcal{E}_{11, \text { em }}^{\text {att rep }}}{\mathcal{T}_{11, \text { em }}^{\text {att rep }}}=-\frac{16 \pi G}{\left(1-\gamma^{-2} g_{11, \text { grav }}\right) c^{5}} .
$$

Proof. By Proposition 5 and 6, we have

$$
\frac{\mathcal{E}_{11, \text { em }}^{a t t r e p}}{\mathcal{T}_{11, \text { em }}^{\text {att rep }}}=\frac{\mp \frac{6 v}{r_{k}^{2} c}}{ \pm \frac{m_{o, Q} v_{Q}}{\frac{4 \pi r^{3}}{3}} \cdot c^{2}}
$$

but as $\frac{1}{r} \rightarrow 0$, one has $\left|\frac{1}{r^{2}}-\frac{1}{r_{K}^{2}}\right| \rightarrow 0$, so that

$$
\frac{\mathcal{E}_{11, \text { em }}^{\text {att rep }}}{\mathcal{T}_{11, \text { em }}^{\text {att }}}=-\frac{8 \pi v r}{c^{3} m_{o, Q} v_{Q}}=-\frac{8 \pi r}{c^{3} m_{o, q}},
$$

where

$$
m_{o, q}=\frac{c^{2}\left(1-\gamma^{2} g_{11, \text { grav }}\right) r}{2 G}
$$

as derived below: First $g_{11 \text {, grav }}$ is the gravitation effect of $q$ on the spacetime metric $g$, but by Remark 3(2)

$$
\begin{aligned}
g_{11, \text { grav }} & =\lambda^{2} \approx \gamma^{2}\left(1-\frac{2 v}{c}\right) \\
& \approx \gamma^{2}\left(1-\frac{2 a t}{c}\right) \\
& =\gamma^{2}\left(1-\frac{2 G m_{o, q}}{r^{2} c} \cdot \frac{r}{c}\right), \text { so } \\
m_{o, q} & ==\frac{c^{2}\left(1-\gamma^{2} g_{11, \text { grav }}\right) r}{2 G} .
\end{aligned}
$$

As such, continuing on Equation (57)

$$
\begin{aligned}
\frac{\mathcal{E}_{11, \text { em }}^{\text {att rep }}}{\mathcal{T}_{11, \text { em }}^{\text {att }}} & =-\frac{8 \pi r}{c^{3} m_{o, q}} \\
& =-\frac{8 \pi}{c^{3}} \cdot \frac{2 G}{c^{2}\left(1-\gamma^{2} g_{11, \text { grav }}\right)} \\
& =-\frac{16 \pi G}{\left(1-\gamma^{-2} g_{11, \text { grav }}\right) c^{5}}
\end{aligned}
$$

Theorem 1

$$
\mathcal{E}_{\mu v, e m}^{a t t r e p}:=R_{\mu v, e m}-\frac{1}{2} R_{e m} \cdot g_{\mu \nu, e m}^{a t t ; r e p}=-\frac{16 \pi G}{\left(1-\gamma_{\text {grav }}^{-2} \cdot g_{11, \text { grav }}\right) c^{5}} \mathcal{T}_{\mu v, e m}^{a t, r e p} .
$$

Proof. (By the above Proposition 7.) 
Remark 4 A distinct feature of gravity is the existence of the principle of equivalence between inertial masses and gravitational masses, so that the two cancel out and the size of the inertial mass does not need to be addressed explicitly, but electromagnetism lacks the same principle and we solved this problem via $\left(1-\gamma_{\text {grav }}^{-2} \cdot g_{11, \text { grav }}\right)$, the greater the inertial mass of the particle being acted upon, the smaller the $g_{11, \text { grav }}$ and hence the greater the denominator of the constant of proportionality. In this connection, we also made a distinct identification of $\mathcal{T}_{11, e m}^{\text {att } r e p}$ with the norm of the Poynting vector $\mathbf{S}$, and as a result, the derived geodesics correspond exactly to the least action by Feynman. As the above showed that a Poynting vector on the right-hand-side of EFE is in direct correspondence with a minimization of the integral of kinetic energy minus potential energy over all trajectories on the left, we see the reasons why any other identifications of $\mathcal{T}_{\mu v, e m}$ have resulted in difficulties in geometrizing electromagnetism. In this regard, our $\mathcal{T}_{11, e m}^{\text {att;ep }}$ has unit joule/(second $\cdot$ meter $\left.^{2}\right)$, representing energy flows in a specific direction across an area of square meter per second, and yet the common identification of $\mathcal{T}_{11, e m}$ with the energy densities has unit joule/ $\left(\right.$ meter $\left.^{3}\right)$, representing stationary energies.

Theorem 2 The set of Einstein Field Equations

$$
\mathcal{E}_{\mu \nu}:=R_{\mu \nu}-\frac{1}{2} R \cdot g_{\mu \nu}=-\frac{8 \pi G}{c^{2}} \mathcal{T}_{\mu v, \text { grav }} \mp \frac{16 \pi G}{\left(1-\gamma^{-2} g_{11, \text { grav }}\right) c^{5}} \mathcal{T}_{\mu \nu, \text { em }}^{\text {attrepul }}
$$

has solutions:

$$
\begin{aligned}
R_{\mu v} & =R_{\mu v, \text { grav }} \pm R_{\mu v, e m}, \\
R & =R_{\text {grav }}+R_{e m}, \\
\text { and } g_{\mu v} & =w_{\text {grav }} \cdot g_{\mu v, \text { grav }} \pm w_{e m} \cdot g_{\mu \mu, e m}^{\text {attrepul }}, \\
\text { with } w_{\text {grav }} & \equiv \frac{R_{\text {grav }}}{R} \text { and } w_{e m} \equiv \frac{R_{e m}}{R} \equiv 1-w_{\text {grav }},
\end{aligned}
$$

where for expository appeal we denote

$$
\begin{aligned}
g_{\mu \nu, e m}^{\text {repul }} & \equiv g_{\mu \nu, e m}^{\text {rep }} \forall \mu \nu \neq 1, g_{11, \text { em }}^{\text {repul }} \equiv-g_{11, e m}^{\text {rep }}=-\lambda_{e m}^{-2} ; \\
\mathcal{T}_{\mu \nu, e m}^{\text {repul }} & \equiv \mathcal{T}_{\mu \nu, e m}^{\text {rep }} \forall \mu \nu \neq 1, \mathcal{T}_{11, \text { em }}^{\text {repul }} \equiv-\mathcal{T}_{11, \text { em }}^{\text {rep }}=\|\overline{\mathbb{S}}(t)\| .
\end{aligned}
$$

Proof. Consider the operation $\mathcal{E}_{\mu v, g r a v} \pm \mathcal{E}_{\mu v, \text { atr }}^{a r e p}$ and denote

$$
\frac{R_{\text {grav }} \cdot g_{\mu v, g r a v}}{R_{\text {grav }}+R_{\text {em }}} \pm \frac{R_{e m} \cdot g_{\mu v, e m}^{\text {attrep }}}{R_{\text {grav }}+R_{\text {em }}}
$$

by $g_{\mu \nu}\left(\equiv w_{\text {grav }} \cdot g_{\mu v, g r a v} \pm w_{e m} \cdot g_{\mu v, e m}^{\text {attrep }}\right)$; we see that the operation of $\mathcal{E}_{\mu v, \text { grav }} \pm \mathcal{E}_{\mu v, e m}^{\text {attrep }}$ is valid if and only if $g_{\mu \nu}$ is forminvariant with respect to measuring geodesics, possessing the same energy interpretations as $g_{\text {grav }}$ and $g_{\text {em }}^{\text {attrep }}$. Here we have:

$$
\begin{aligned}
& (1,0,0,0) \circ\left(w_{\text {grav }} \cdot g_{\text {grav }}+w_{\text {em }} \cdot g_{\text {em }}^{\text {att }}\right) \circ\left(-1, \mathbf{V}_{x}, \mathbf{V}_{y}, \mathbf{V}_{z}\right)^{T} \\
& (\text { cf. Equation }(17)) \\
= & w_{\text {grav }} \cdot\left(-1-2 \cdot\left(\frac{K E_{\text {grav }}}{R E}\right)+2 \cdot\left(\frac{P E_{\text {grav }}}{R E}\right)\right) \\
& +w_{\text {em }} \cdot\left(-1-2 \cdot\left(\frac{K E_{\text {em }}^{\text {att }}}{R E}\right)+2 \cdot\left(\frac{P E_{\text {em }}^{\text {att }}}{R E}\right)\right) \\
\equiv & -1-\frac{2 K E_{\text {gravem }}^{\text {att }}}{R E}+\frac{2 P E_{\text {gravem }}^{\text {att }}}{R E},
\end{aligned}
$$

where

$$
\begin{aligned}
K E_{\text {gravem }}^{\text {att }} & \equiv w_{\text {grav }} \cdot K E_{\text {grav }}+w_{\text {em }} \cdot K E_{\text {em }}^{\text {att }}, \text { and } \\
P E_{\text {gravem }}^{\text {att }} & \equiv w_{\text {grav }} \cdot P E_{\text {grav }}+w_{\text {em }} \cdot P E_{\text {em }}^{\text {att }} .
\end{aligned}
$$

Now since

$$
\left(-R_{11, e m}\right)-\frac{1}{2} R \cdot g_{11, \text { em }}^{\text {repul }}=\frac{-16 \pi G}{\left(1-\gamma^{-2} g_{11, \text { grav }}\right) c^{5}} \mathcal{T}_{11, \text { em }}^{\text {repul }}
$$


and

$$
\begin{aligned}
& (1,0,0,0) \circ g_{\text {em }}^{\text {repul }} \circ\left(-1, \mathbf{V}_{x}, \mathbf{V}_{y}, \mathbf{V}_{z}\right)^{T} \\
\equiv & (1,0,0,0) \circ g_{\text {em }}^{\text {rep }} \circ\left(1, \mathbf{V}_{x}, \mathbf{V}_{y}, \mathbf{V}_{z}\right)^{T}
\end{aligned}
$$

we have

$$
\begin{aligned}
& (1,0,0,0) \circ\left(w_{\text {grav }} \cdot g_{\text {grav }}-w_{\text {em }} \cdot g_{\text {em }}^{\text {repul }}\right) \circ\left(-1, \mathbf{V}_{x}, \mathbf{V}_{y}, \mathbf{V}_{z}\right)^{T} \\
= & w_{\text {grav }} \cdot\left(-1-2 \cdot\left(\frac{K E_{\text {grav }}}{R E}\right)+2 \cdot\left(\frac{P E_{\text {grav }}}{R E}\right)\right) \\
& -w_{\text {em }} \cdot\left(1-2 \cdot\left(\frac{K E_{\text {em }}^{\text {rep }}}{R E}\right)+2 \cdot\left(\frac{P E_{\text {em }}^{\text {rep }}}{R E}\right)\right) \\
\equiv & -1-\frac{2 K E_{\text {gravem }}^{\text {rep }}}{R E}+\frac{2 P E_{\text {gravem }}^{\text {rep }}}{R E},
\end{aligned}
$$

where

$$
\begin{aligned}
& K E_{\text {gravem }}^{\text {rep }} \equiv w_{\text {grav }} \cdot K E_{\text {grav }}-w_{\text {em }} \cdot K E_{\text {em }}^{r e p}, \text { and } \\
& P E_{\text {gravem }}^{\text {rep }} \equiv w_{\text {grav }} \cdot P E_{\text {grav }}-w_{\text {em }} \cdot P E_{\text {em }}^{r e p} .
\end{aligned}
$$

Consequently, $g_{\mu \nu}=w_{\text {grav }} \cdot g_{\mu v, \text { grav }} \pm w_{e m} \cdot g_{\mu v, \text {,em }}^{\text {attrepul }}$ is form-invariant in measuring geodesics, with identical interpretations of energies to that of $g_{\mu v, g r a v}$ and $g_{\mu v, e m}^{a t t r e p}$. I.e.,

$$
\mathcal{E}:=\mathcal{E}_{\text {grav }} \pm \mathcal{E}_{\text {em }}^{\text {attrep }}=-\frac{8 \pi G}{c^{2}} \mathcal{T}_{\text {grav }} \mp \frac{16 \pi G}{\left(1-\gamma^{\mp 2} g_{11, \text { grav }}\right) c^{5}} \mathcal{T}_{\text {em }}^{\text {att;repul }}
$$

results in a metric $g_{\mu v}$ that renders

$$
g_{1} \circ(-1, \mathbf{V})^{T}=-1-\frac{2 K E_{\text {gravem }}}{R E}+\frac{2 P E_{\text {gravem }}}{R E} .
$$

\subsection{Integration of the Nuclear Strong Force with Gravity}

Most recently we showed that (Light, 2019): (1) quarks combine themselves by superpositions of coincidental fields, hence inseparable, and (2) proton $P$ has a mini black hole of radius $10^{-15}$ meters, attracting a neutron $n$ with $g_{11} \approx 10^{-20}$ by the extended EFE, so that

$$
\frac{G \cdot 10^{10} m_{P} \cdot 10^{10} m_{n}}{\left(10^{-10} r\right)^{2}}=10^{40} \cdot \frac{G m_{P} m_{n}}{r^{2}} .
$$

As such, the nuclear strong force is a manifestation of gravity. For the purpose of this paper we will give a sketch of the proof of $g_{11} \approx 10^{-20}$ by the extended EFE.

A rest proton has its mass $\approx 1.67 \times 10^{-27} \mathrm{~kg}$ with Lorentz factor $\gamma=1$, so that

$$
\begin{aligned}
1-\gamma^{-2} g_{11, \text { grav }} & =1-g_{11, \text { grav }} \\
& =1-\left(1-\frac{G \times 1.67 \times 10^{-27}}{r c^{2}}\right) \\
& =\frac{G \times 1.67 \times 10^{-27}}{r c^{2}} ;
\end{aligned}
$$

suppose we substitute

$$
r=10^{-15} \text { e meters, } e \approx 2.7,
$$

into the above equation; then we have

$$
1-\gamma^{-2} g_{11, \text { grav }}=\frac{G \times 1.67 \times 10^{-27}}{10^{-15} e \times 9 \times 10^{16}} \approx 0.46 \times 10^{-39} .
$$


Since

$$
\begin{aligned}
\left(\frac{\mathcal{T}_{11, \text { em }}^{\text {att }}}{\mathcal{T}_{11}, \text { grav }}\right) & =\frac{1 \cdot J /\left(\mathrm{sm}^{2}\right)}{1 \cdot \mathrm{kg} / \mathrm{m}^{3}} \\
& =\frac{1 \cdot \mathrm{kgc} /\left(\mathrm{c}^{-1} \mathrm{~m}^{3}\right)}{1 \cdot \mathrm{kg} / \mathrm{m}^{3}} \\
& =c^{3},
\end{aligned}
$$

we have

$$
\begin{aligned}
& \frac{16 \pi G \mathcal{T}_{11, \text { at }}^{\text {att }}}{0.46 \times 10^{-39} c^{5}} \cdot \frac{c^{2}}{8 \pi G \mathcal{T}_{11}, \text { grav }} \\
= & \frac{2}{0.46} \times 10^{39}=4.35 \times 10^{39} \\
= & \frac{\|E M(P)\|}{\|\operatorname{Grav}(P)\|} .
\end{aligned}
$$

Consequently the Schwarzschild radius of proton is $4.35 \times 10^{39}$ times the radius as based on its (gravitational) mass alone and

$$
\begin{aligned}
r_{S c h}(P) & \approx r_{\text {Sch,grav }}(P) \times 4.35 \times 10^{39} \\
& =1.24 \times 10^{-54} \times 4.35 \times 10^{39} \\
& \approx 10^{-15}(\mathrm{~m}) .
\end{aligned}
$$

Therefore,

$$
\begin{aligned}
g_{11} & =\left(\frac{t_{0}^{[2]}}{t_{0}^{[1]}}\right)^{2}=1-\frac{r_{S c h}(p)}{r} \\
& =1-\frac{r_{S c h}(P)}{r_{S c h}(P)+\frac{\pi}{6} \frac{\lambda_{P}}{2}} \\
& \approx \frac{1.3 \times 10^{-35}}{10^{-15}} \\
& =1.3 \times 10^{-20} .
\end{aligned}
$$

where we calculate the gravitational strength of $P$ outside its Schwarzschild radius $r_{S c h}(P)$ by our assumed least indivisible unit of length, $\frac{\pi}{6} \frac{\lambda_{P} \text { (Planck length) }}{2}$.

\subsection{Integration of the Nuclear Weak Force with Gravity}

In the above analysis we noticed that $n$, not possessing a mini black hole due to having no electric charge, is fundamentally different from $P$ (cf. Bigazzi, \& Niro, 2018, for isospin-breaking); for example, while the radius of $P$ is $10^{-15} e$ meters, that of $n$ is $10^{-15}$ meters, smaller. Since in our hypothesized diagonal 4-manifold $\mathcal{M}^{[3]}$ of the Universe (cf. Brandenberger, et al., 2018, and Sengupta, 2017, for other constructs of a dual universe not based on the particle-wave duality), black holes are intersections of the visible particle universe $\mathcal{M}^{[1]}$ and the invisible wave universe $\mathcal{M}^{[2]}, P$ being embedded in its own mini black hole does not vanish from $\mathcal{M}^{[1]}$, but $n$ without a mini black hole vanishes from $\mathcal{M}^{[1]}$ (cf. Barducci, 2018, for "disappearing into the darkness"), which motivates the following analysis.

\subsubsection{Lifetime of Neutron}

Proposition 8 Assume that the distribution of energy E over (particle p, EMW $(p)$ ) is $\left(\frac{3}{4} E, \frac{1}{4} E\right)$. Then $E=1.6 \hat{E}$, where $\hat{E} \equiv$ the laboratory-measured energy of $p$.

Proof. Previously we derived the metric tensor $g^{[3]}$ of $\mathcal{M}^{[3]}$ to be

$$
\begin{aligned}
g_{11}^{[3]} & =\frac{G^{[2]}}{G^{[1]}+G^{[2]}} g_{11}^{[1]}+\frac{G^{[1]}}{G^{[1]}+G^{[2]}} g_{11}^{[2]}, \text { with } \\
\frac{G^{[1]} G^{[2]}}{G^{[1]}+G^{[2]}} & =G^{[3]} .
\end{aligned}
$$


Therefore,

$$
\begin{aligned}
& m^{[3]} \mathbf{a}^{[3]}=-\left[\left(\frac{G^{[2]}}{G^{[1]}+G^{[2]}}\right)\left(\frac{G^{[1]} M^{[1]} m^{[1]}}{r^{2}}\right)\right. \\
& \left.+\left(\frac{G^{[1]}}{G^{[1]}+G^{[2]}}\right)\left(\frac{G^{[2]} M^{[2]} m^{[2]}}{r^{2}}\right)\right] \cdot \frac{\mathbf{r}}{r} ;
\end{aligned}
$$

hence

$$
\begin{aligned}
\mathbf{a}^{[3]} & =-\frac{G^{[3]} M^{[3]}}{r^{2}} \cdot\left(\frac{M^{[1]}}{M^{[3]}} \frac{m^{[1]}}{m^{[3]}}+\frac{M^{[2]}}{M^{[3]}} \frac{m^{[2]}}{m^{[3]}}\right) \cdot \frac{\mathbf{r}}{r} \\
& =-\frac{G^{[3]} M^{[3]}}{r^{2}} \cdot\left(\frac{9}{16}+\frac{1}{16}\right) \cdot \frac{\mathbf{r}}{r} \\
& \equiv-\frac{G^{[3]} \hat{M}}{r^{2}} \cdot \frac{\mathbf{r}}{r}, \text { where } \\
M^{[3]} & =1.6 \hat{M} .
\end{aligned}
$$

Corollary 1 Any particle $p$ that has its EMW ( $p$ ) a union of two semi-circular rotations presents itself as ( $p, E M W(p))$ for a duration of $\frac{5}{8} \frac{\lambda}{c}$ and $(0, E M W(p))$ for a duration of $\frac{3}{8} \frac{\lambda}{c}$, where $\lambda \equiv$ the wave length of $E M W(p)$ (to define such particles as "single-cycle particles").

Proof. $E=1.6 \hat{E}=1.6 h v=h \cdot \frac{c}{\frac{5}{8} \lambda}$.

Remark 5 The laboratory-measured energy of a single-cycle particle $p$ is $\hat{E}$ owing to the existence of $(p, \lambda(p))$. Since wave is probability of no energy in the quantum formulation (which contains the formula $\hat{E}=h v$ ), $p$ must carry the entire energy $E=1.6 \hat{E}$ that would enjoy a higher frequency $1.6 v$, but the measured frequency is (still) $v$; therefore, $p$ exists (only) in $\left[0, \frac{5}{8}\right] \lambda$. That is, the fact that $p$ carries a wave length $\lambda(p)$ does not imply $p$ exists throughout $[0,1] \lambda$, as evidenced in, say, quantum tunneling. Equivalently, if $p$ lasted for $[0,1] \lambda$, then one would have $E=h \cdot \frac{c}{\lambda}=h v=\hat{E}$, contradicting $E=1.6 \hat{E}$.

Remark 6 Single-cycle particles include electron, neutrino, proton, neutron, photon, and any hadrons that are made up of $u$ and/or $d$.

Proposition 9 Assume that in the motion of $\operatorname{EMW}(n): W \rightarrow N \rightarrow E \rightarrow N \rightarrow W$, the stopping time at $W$ and $E$ is $10^{-24}$ seconds; then $n$ in isolation has a lifetime about 900 seconds.

Proof. By the above Remark 3 (2) the ratio of two proper times is the eigenvalue $\lambda$ of the Lorentz transformation,

$$
\lambda=\sqrt{\frac{c-v}{c+v}} .
$$

Set the proper time $t_{0}^{[2]}$ to be that of $E M W(n)$ and $t_{0}^{[1]}$ to be that of the laboratory frame. Then

$$
\frac{t_{0}^{[2]}}{t_{0}^{[1]}}=\sqrt{\frac{c-v}{c+v}} .
$$

Since by Corollary 1

$$
\begin{aligned}
t_{0}^{[2]} & =\frac{5}{8} \frac{\lambda}{c}=\frac{5}{8} v=\frac{5}{8} \frac{h}{E_{[2]}} \text { and } \\
E_{[2]} & =E_{[1]} \sqrt{\frac{c-v}{c+v}}
\end{aligned}
$$

(in analogy with $m_{0}=m \gamma^{-1}$, but the relation here is that of $\lambda=\gamma\left(1-\frac{v}{c}\right)$, not of the Lorentz factor $\gamma$ ), one has

$$
\begin{aligned}
t_{0}^{[1]} & =900 \text { seconds if and only if } \\
\frac{5}{8} \frac{h}{E_{[1]} \sqrt{\frac{c-v}{c+v}}} & =\sqrt{\frac{c-v}{c+v}} \cdot 900 .
\end{aligned}
$$


Substituting the rest mass $m_{0}(n)=1.68 \times 10^{-27} \mathrm{~kg}$ into $E_{[1]}=m_{0}(n) c^{2}$, one has

$$
\frac{6.63 \times 10^{-34}}{1.6 \times 1.68 \times 10^{-27} \times 9 \times 10^{16}}=\frac{c-v}{c+v} \cdot 900 \text {, }
$$

i.e.,

$$
\frac{c-v}{c+v} \approx 3 \times 10^{-27}
$$

implying that $v \lesssim c$ and

$$
1-\frac{v}{c} \approx 6 \times 10^{-27}
$$

this reduction of speed in $E M W(n)$ by a factor of $6 \times 10^{-27}$ implies a prolongation of $n$ to finish $\frac{5}{8} \lambda$ by (about) the same factor (as in $(1-\epsilon)^{-1} \approx 1+\epsilon$ ). Thus in the laboratory frame, $E M W(n)$ stops at the intersection points $W$ and $E$ for about

$$
900 \times 6 \times 10^{-27}=5.4 \times 10^{-24} \text { seconds. }
$$

Remark 7 The lifetime of $n$ in its own frame is then $900 \sqrt{3 \times 10^{-27}} \approx 5 \times 10^{-11}$ seconds (cf. Leontaris, \& Vergados, 2019 , for recent interest in the neutron lifetime puzzle).

2.3.2 Neutron Decay

Proposition 10 Assume that the Higgs particle h has a representation

$$
\begin{aligned}
h & =2 \gamma_{L}+\gamma_{R}+\gamma_{i L}, \text { where } \\
\gamma_{L} & : \quad=W \rightarrow N \rightarrow E \rightarrow S \rightarrow W, \\
\gamma_{R} & : \quad=W \rightarrow S \rightarrow E \rightarrow N \rightarrow W, \text { and } \\
\gamma_{i L} & : \quad=E \rightarrow T \rightarrow W \rightarrow B \rightarrow E .
\end{aligned}
$$

Then

$$
\begin{aligned}
h & =W^{-}+W^{+}, \text {where } \\
W^{-} & =W \rightarrow N \rightarrow E \rightarrow T \rightarrow W^{*(\text { branch point })} \\
& \rightarrow S \rightarrow E \rightarrow S \rightarrow W \\
( & =e^{-}+\tilde{v} \text { when at } W^{*} \text { the flow splits into } \\
e^{-} & =W \rightarrow N \rightarrow E \rightarrow T \rightarrow W \text { and } \\
\tilde{v} & =W \rightarrow S \rightarrow E \rightarrow S \rightarrow W)
\end{aligned}
$$

and

$$
\begin{aligned}
W^{+} & =E \rightarrow S \rightarrow W \rightarrow B \rightarrow E^{*(\text { branch point })} \\
& \rightarrow N \rightarrow W \rightarrow N \rightarrow E \\
( & =e^{+}+v \text { when at } E^{*} \text { the flow splits into } \\
e^{+} & =E \rightarrow S \rightarrow W \rightarrow B \rightarrow E \text { and } \\
v & =E \rightarrow N \rightarrow W \rightarrow N \rightarrow E) .
\end{aligned}
$$

Proof. We index the flows to keep track of the re-combination of waves:

$$
\begin{aligned}
W^{-} & =W_{1} \rightarrow N_{2} \rightarrow E_{3} \rightarrow T_{4} \rightarrow W_{5} \\
& \rightarrow S_{6} \rightarrow E_{7} \rightarrow S_{8} \rightarrow W_{9} \\
& =\left(W_{1} \rightarrow N_{2} \rightarrow E_{3}\right) \subset \gamma_{L} \\
\left(\rightarrow T_{4} \rightarrow W_{5}\right) & \subset \gamma_{i L} \\
\left(\rightarrow S_{6} \rightarrow E_{7}\right) & \subset \gamma_{R} \\
\left(\rightarrow S_{8} \rightarrow W_{9}\right) & \subset \gamma_{L} ;
\end{aligned}
$$




$$
\begin{aligned}
W^{+} & =E_{i} \rightarrow S_{i i} \rightarrow W_{i i i} \rightarrow B_{i v} \rightarrow E_{v} \\
& \rightarrow N_{v i} \rightarrow W_{v i i} \rightarrow N_{v i i i} \rightarrow E_{i x} \\
& =\left(E_{i} \rightarrow S_{i i} \rightarrow W_{i i i}\right) \subset \gamma_{L} \\
\left(\rightarrow B_{i v} \rightarrow E_{v}\right) & \subset \gamma_{i L} \\
\left(\rightarrow N_{v i} \rightarrow W_{v i i}\right) & \subset \gamma_{R} \\
\left(\rightarrow N_{v i i i} \rightarrow E_{i x}\right) & \subset \gamma_{L} .
\end{aligned}
$$

Proposition 11 Set $n=E \rightarrow T \rightarrow W \rightarrow T \rightarrow E$; then $W^{-}+n=e^{-}+\tilde{v}+P$.

Proof.

$$
\begin{aligned}
W^{-}+n= & \left(W \rightarrow N \rightarrow E \rightarrow T \rightarrow \mathbf{W}_{\text {branch for } e^{-}}\right) \text {as } e^{-} \\
& +\left(\mathbf{W}_{\text {branch for } \tilde{v}} \rightarrow S \rightarrow \mathbf{E}_{\text {branch for } \tilde{v}} \rightarrow S \rightarrow W\right) \text { as } \tilde{v} \\
& +\left[\mathbf{W} \rightarrow S \rightarrow \mathbf{E}_{\text {branch for } P}(\rightarrow T \rightarrow W \text { from } n)\right] \text { as } P .
\end{aligned}
$$

Remark 8 Weak decay has many practical implications, for example, nuclear reactor operations (Gebre, \& Surukuchi, 2018). Our graph-theory approach may serve as an added tool in quantum field theories.

\section{Discussion}

(1) Every single-cycle particle $(p, \lambda(p))$ exists in $\mathcal{M}^{[3]}$ for a duration of $\frac{5}{8} \frac{\lambda}{c}$; then it becomes $(0, \lambda(p))$ in $\mathbf{B} \subset \mathcal{M}^{[2]}$ for the remaining $\frac{3}{8} \frac{\lambda}{c}$. This process repeats itself if $p$ has a mini black hole; otherwise, $\lambda(p)$ can combine with other waves to form new particle(s) (for recent studies on beta decay, see, e.g., Deppisch, et al., 2018).

(2) Both the strong and the weak nuclear forces can be explained by General Relativity via the metric tensor $g_{11}$ which relates two proper spacetimes.

(3) As EFE also explains electromagnetism, gravity remains as the sole fundamental force as based on our geometry of diagonal 4-manifold. We remark that the basic spacetime hypotheses determine the rest of Physics (cf. Minazzoli, 2018).

\section{References}

Barducci, D., Fabbrichesi, M., \& Gabrielli, E. (2018). Neutral hadrons disappearing into the darkness. Physical Review D, 98, 035049 (1-9). https://doi.org/10.1103/PhysRevD.98.035049

Bigazzi, F., \& Niro, P. (2018). Neutron-proton mass difference from gauge/gravity duality. Physical Review D, 98,046004 (1-12). https://doi.org/10.1103/PhysRevD.98.046004

Brandenberger, R., Costa, R., Franzmann, G., \& Weltman, A. (2018). Dual spacetime and nonsingular string cosmology. Physical Review D, 98, 063521(1-7). https://doi.org/10.1103/PhysRevD.98.063521

Deppisch, F. F., Graf, L., Harz, J., \& Huang, W-C. (2018). Neutrinoless double beta decay and the baryon asymmetry of the Universe. Physical Review D, 98, 055029 (1-28). https://doi.org/10.1103/PhysRevD.98.055029

Gebre, Y., Littlejohn, B. R., \& Surukuchi, P. T. (2018). Prospects for improved understanding of isotopic reactor antineutrino fluxes. Physical Review D, 97, 013003 (1-12). https://doi.org/10.1103/PhysRevD.97.013003

Kamada, K. (2018). Return of grand unified theory baryogenesis: Source of helical hypermagnetic fields for the baryon asymmetry of the universe. Physical Review D, 97, 103506(1-5). https://doi.org/10.1103/PhysRevD.97.103506

Leontaris, G. K., \& Vergados, J. D. (2019). n-n̄ oscillations and the neutron lifetime. Physical Review D, 99,015010 (1-11). https://doi.org/10.1103/PhysRevD.99.015010

Light, G. L. (2019). Quantum mechanics by general relativity. Applied Physics Research, 11(2), 1-9. https://doi.org/10.5 539/apr.v11n2p1

Minazzoli, O. (2018). Rethinking the link between matter and geometry. Physical Review D, 98, 124020 (1-5). https://doi.org/10.1103/PhysRevD.98.124020

Sengupta, S. (2017). Spacetime-bridge solutions in vacuum gravity. Physical Review D, 96, 104031 (1-6). https://doi.org/10.1103/PhysRevD.96.104031

\section{Copyrights}

Copyright for this article is retained by the author(s), with first publication rights granted to the journal.

This is an open-access article distributed under the terms and conditions of the Creative Commons Attribution license (http://creativecommons.org/licenses/by/4.0/). 